International Journal of Web \& Semantic Technology (IJWesT) Vol.3, No.1, January 2012

\title{
A COMBinAtional APPROACH OF GIS AND SOA For PERFORMANCE IMPROVEMENT OF ORGANIZATION
}

\author{
Mortaza Saleh $^{1}$, Tahere Yaghoobi ${ }^{2}$ and Ahmad Faraahi ${ }^{3}$ \\ ${ }^{1}$ Department of Computer Engineering and Information Technology, Payame Noor \\ University, Tehran \\ Saleh.Mortaza@Gmail.com \\ ${ }^{2}$ Department of Computer Engineering and Information Technology, Payame Noor \\ University, Isfahan \\ T.Yaghoobiepnu.ac.ir \\ ${ }^{3}$ Department of Computer Engineering and Information Technology, Payame Noor \\ University, Tehran \\ Afaraahi@Pnu.ac.ir
}

\begin{abstract}
A GIS (Geographic Information System), is a system designed to capture, store, manipulate, analyze, manage, and present all types of geographically referenced data. In other words, GIS is the merging of statistical analysis, database technology and cartography that can be integrated into any enterprise information system framework. But despite all these advantages, geographic information systems that used in organizational structures, is faced with some problems such as lack of interoperability, business alignment, and agility.

This paper first analyzes the connotation, model structure and main features of GIS and Service Oriented Architecture (SOA). Then we propose a service oriented GIS platform which uses SOA concepts as the basic architectural concept and employs OGC (Open Geospatial Consortium) standards to support GIS concerns in the organization. Our combinational approach, using features such as loose coupling, integrity, independence and etc., can overcome the most of GIS problems such as lack of interoperability, business alignment, and agility.
\end{abstract}

\section{Keywords}

Geographic Information System (GIS); Service Oriented Architecture (SOA); Service; Application Service; Business Service; GIS Service; Platform.

\section{INTRODUCTION}

Nowadays having access to correct, accurate, updated and reliable information is one of the most important factors of decision making in all aspects of societies. Regarding this concept, the definition of information in various societies was restricted to attribute data and statistical data until several years ago. Therefore, collecting, keeping and updating those information for decision making for the daily affairs, was both time consuming and expensive. But after the passage of little time, research conducted by United Nation and international committee of

DOI : 10.5121/ijwest.2012.3102 
geomatics revealed that more than $80 \%$ of exchanged information in the organizational settings has geospatial characteristic [1]. Meanwhile, the above mentioned organization provided a definition for such information which is called spatial data. Here is the definition: spatial data are those data which are related to the features on the ground. Therefore it was identified that spatial data include other usual and conceivable data such as attributed data and statistical data and etc. in the form of charted data that are related to the main feature $[2,3]$.

After one decade, all organizations embarked on collection and producing spatial data by surveying, photogrammetry, remote sensing, etc. but due to the lack of a unified and well defined procedure, using the data was so difficult that this process was taken place manually. Therefore, as data increased, decisions could not be taken manually by those who were in change [4]. As a result, in the early 1980 especial kind of information systems emerged with the title of Geographic Information System (GIS). This information system utilized some data, information, rules, procedures, and computers and was able to analyze mentioned data and offer much more precise ways for decision making by combining spatial and attribute data $[5,6]$.

As time elapsed some problems gradually appeared. Because several isolated parts inside the organizations were formed and each part worked independently. They constituted systems and data without knowing anything about the works of other parts. Therefore the organizations faced with a huge cost and also wasted time. Moreover, data produced in this method are in various formats, systems, and accuracy and lack metadata. According to the latest finding, using Geodatabase with the especial architecture of spatial data can solve this problem $[2,3]$.

In spite of its advantages, this type of architecture, like other newly developed systems, has some disadvantages such as lack of interoperability, business alignment, and agility. These disadvantages decrease the output of the system and waste lot of time as well $[7,8]$. On the other hand, when an organization offers and receives services, it becomes necessary for that organization to develop a strategic platform of technology which can enable all system of inside or outside the organization to offer and receive service and also satisfy the demand of users [9, $10]$.

According to these explanations, service oriented architecture can be considered as a new and developing method for making distributed application which is able to solve the big problem of disunity among the organizational systems and also by providing software application paves the way for better interoperation between business and information technology. Consequently, it seems that combining service oriented architecture with standards of Open Geospatial Consortium (OGC) can be an effective solution for the problems of geographic information system. OGC standards are some technical documents which exactly elaborate on encodings and connections. The main purpose of developing these standards is solving the problems of interoperability among GIS software [11]. To satisfy this concern, in this paper, SOA is defined as the best practice of the architectural concepts for increasing business alignment in the enterprise applications. Then we propose a service-oriented GIS platform based on OGC standards and in order to business enterprise. This study insists on developing GIS software applications on the ground of SOA concepts and OGC standards.

This paper is organized as follows: related works and our motivation are reviewed in section 2 . Section 3 is focused on service oriented architecture and its general structure. In section 4 we propose our approach which contains our desired strategy, methodology and our proposed platform. A conclusion and an outlook on future research in this area close the body of this paper. 
International Journal of Web \& Semantic Technology (IJWesT) Vol.3, No.1, January 2012

\section{RELATED WORKS AND MOTIVATION}

Despite all the above advantages, GIS applications have greatly suffered both from lack of interoperability between internal software components and lack of alignment with real enterprise concerns [12]. It is worth mentioning that many efforts have been done in this context, but there is not a clear solution. Most other previous related works have the common goal of making GIS applications more common and open. Some efforts worry about applying component oriented approaches to GIS application development $[13,14]$. Some of them focused on OGC capabilities through utilizing OGC web services (OWSs) in their architecture [15, 16]. And some others focused on SOA concepts and patterns implementation without considering OGC standards in the architecture [17-19]. What is clear is that previous efforts and related works have not strictly focused on the enterprise and GIS concerns at the same time. In this context and through our combinational approach, enterprise and GIS concerns are considered together.

\section{SERVICE ORIENTED ARCHITECTURE}

Increasingly complex requirements of IT systems and the need to adopt with required changes have attracted the organizations to focus on SOA to align their IT with their business processes. SOA as the best practice for increasing business alignment in the enterprise applications, comprise valuable characteristics like loose coupling, reusability, abstraction, agility, and etc., that result simple software development and time and cost efficient [20-23].

As reported by [24], service oriented architecture can be viewed from three different perspectives:

- The business perspective: Set of provided services by an organization to its stakeholders;

- The architecture perspective: An architectural view which consists of a service provider, a requester, and a service discovery;

- The implementation perspective: Set of standards, tools, and technologies, such as web services.

According to this classification, essentially, service oriented architecture is a collection of services that communicate with each other $[25,26]$. The communication can involve either simple data passing or it could involve two or more services coordinating some activity. Here, a service is a function that is well-defined, self-contained, does not depend on the context or state of other services and from the organizational perspective, have become an impressive factor for growth and the creation of jobs as $93 \%$ of the new jobs created in the U.S. between 1970 and 2000 are jobs in services [27]. SOA services can be easily combined and integrated with other services to satisfy business processes.

On the other hand, according to the information technology perspective, logical organization, divided in two major sections: business logic and application logic. Using these logics, organizational processes and their appropriate services are created. These services can encapsulate both logics. Figure 1 shows the service interface layer between the information layers of organization. By layering services and their abstraction, service-oriented features can be achieved significantly and so, organization speed will be higher. These layers are application, business, and orchestration.

Application services layer, are useful services with reusability that usually are implemented in two models: "utility services" and "wrapper services". Business services layer, are the controllers 
that combine application services for implement their business logic. Orchestration services layer include one or more process services that combine application services and business services according to the logic and business rules; This is a coordinator. More details are mentioned in [28].

In the following, we use service oriented architecture and its characteristics to present our related strategy, methodology, and finally to propose our slightly platform.

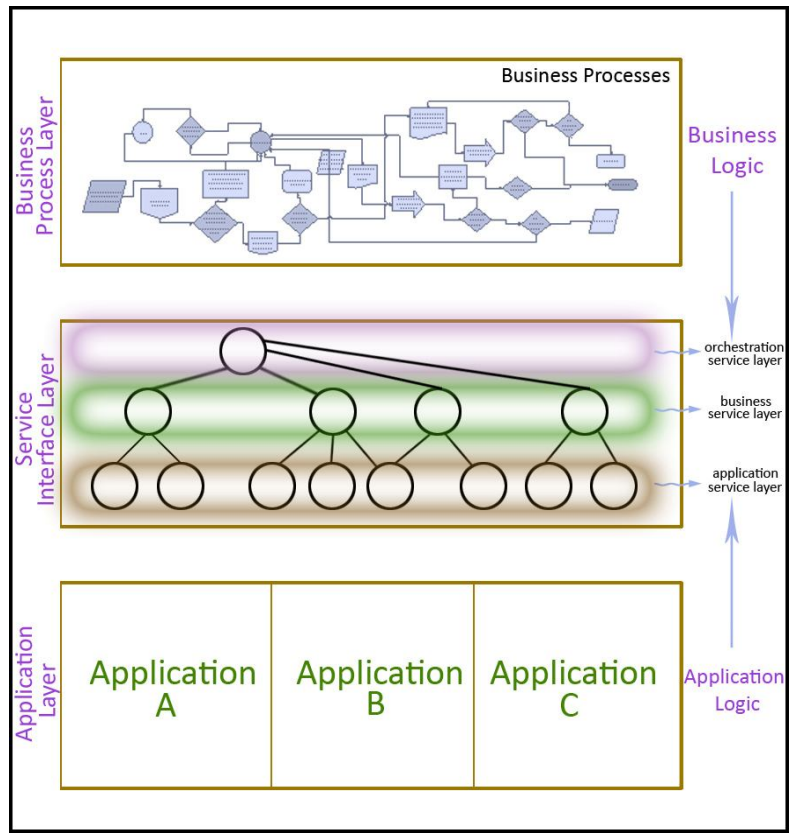

Figure 1. Service Oriented Architecture in General View

\section{OUR PROPOSED APPROACH}

\subsection{STRATEGY}

In general, three strategies are common in software engineering: Top-down, Bottom-up and Agile strategy $[26,29]$ that we selected agile strategy in this work.

\subsubsection{TOP-DOWN}

This strategy supports three layers of services that mentioned in previous section. In this strategy, detailed definition of business models presents before modeling the service boundaries. Although the best quality of service oriented architecture is offered in top-down strategy, but a considerable amount of time and work is allocated to the analysis.

\subsection{2 ВотTOM-UP}

In this strategy, creating services considered to be as the base for achieve application-centric requirements. Integration is the main work for bottom-up design. Using this strategy, organizations can add services to their application environments easily. 


\subsubsection{AGILE}

We selected agile strategy due to include benefits of both top-down and bottom-up strategies. This strategy combines benefits of both top-down and bottom-up approaches to meet the immediate requirements without compromising the integrity of the enterprise business model and characteristics of service oriented architecture. Figure 2 shows this strategy steps.

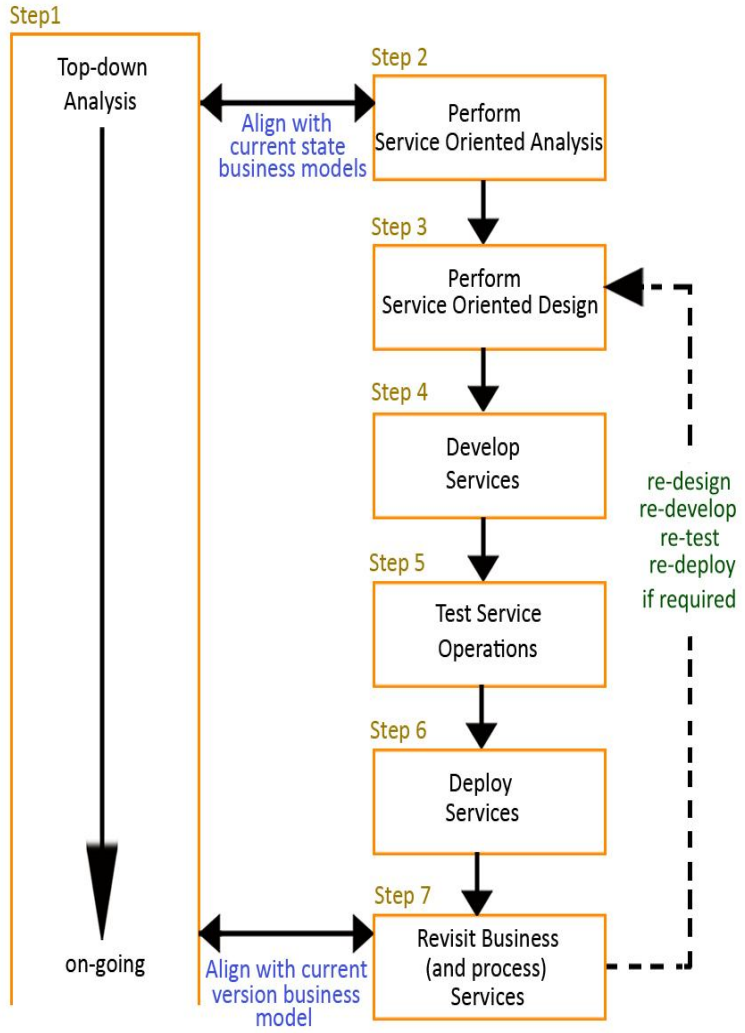

Figure 2. Agile Strategy Steps

\subsection{METHODOLOGY}

Nowadays having a clear methodology is often deemed important especially in the computer science. A methodology is a body of knowledge comprising the principles, guidelines, best practices, methods, and processes relating to a particular discipline such as interaction design or user research. In most software engineering projects, a methodology is usually presented not as a description but as a prescription or a recommendation that projects should follow the generalized task structure; It is a much broader concept than a process and can increase the quality control by ensuring that the procedures are consistent.

As shown in figure 3, our methodology consists of seven phases. These phases, show different stages of service oriented projects. Due to specific nature of service oriented architecture, two primary phases of this methodology are examined here. 


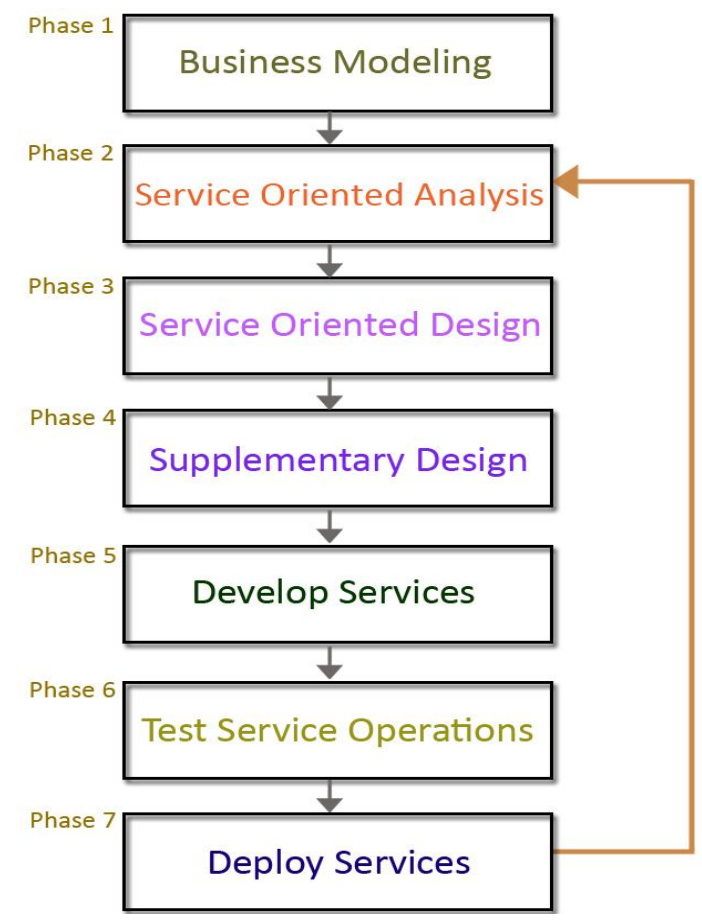

Figure 3. Methodology Phases

\subsubsection{BUSINESS MODELING}

In the first phase of the business modeling, we define interactions between a role and / or a system to achieve a goal through use cases. Then, each of them should be realized. Use case realization represents how a use case will be implemented in terms of collaborating objects. This can take various forms. It can include, for example, a textual description (a document), class diagrams of subsystems and participating classes, and interaction diagrams (sequence and communication diagrams) that illustrate the flow of interactions between class and sample subsystem.

Due to better management, use case realization is separated from use case. This isolation allows the use cases to be managed separately from their realizations. This is particularly important for larger projects, or chains of systems where the same use cases can be designed differently in various products within the product chain. For larger projects, separating the use case and its realization allows changes to the design of the use case without affecting the baselined use case itself.

Next step identifies the events and business workers. Business event is an extraordinary occurrence or any observable occurrence that has emphasis to a business. A business worker is a class that represents an abstraction of a human that acts within the system. Business worker manipulates business entities while participating in business use case realizations and interacts with other business workers. As shown in figure 4, business modeling is output of these three stages and useful in identifying business architecture, business rules, and business goals. 


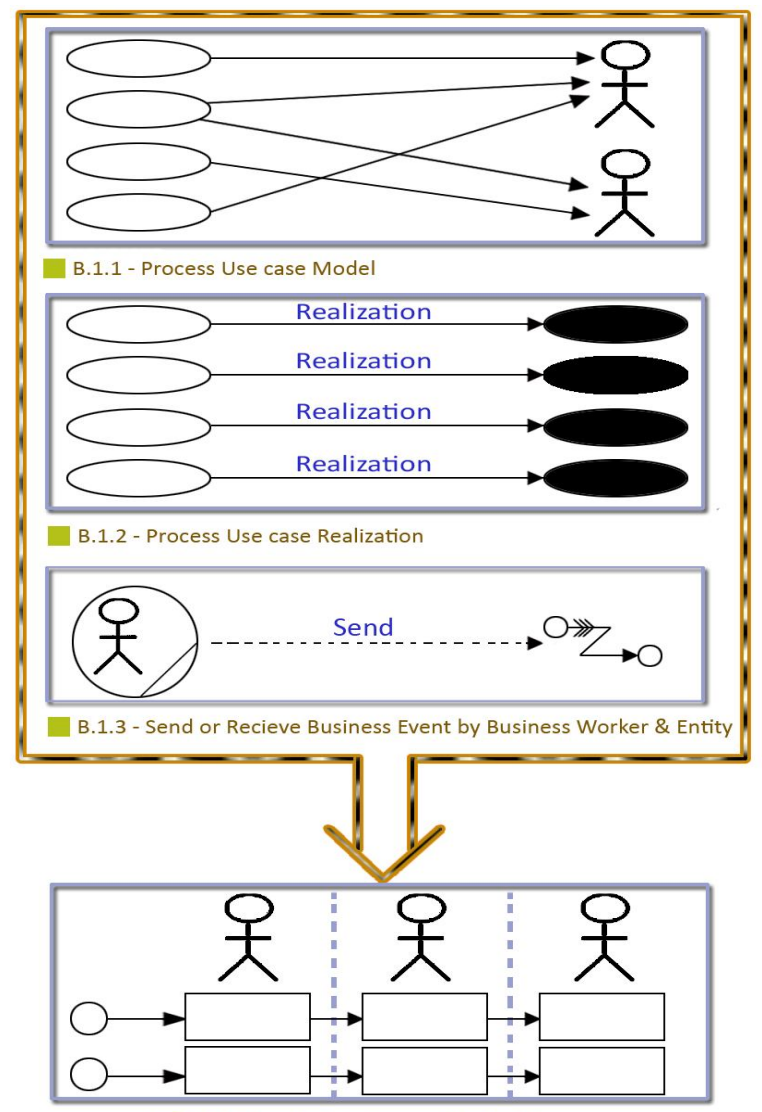

B.1.Final - Business Model Process

Figure 4. Business Modeling

\subsubsection{SERVICE ORIENTED ANALYSIS}

The second phase of our methodology has eight steps. First step is related to the requirements; business requirements have a direct affiliation with business activities and used to communicate how a new or existing business process should work. In IT projects, business requirement is often a precursor to designing and building a new business application / system, or changing an existing one, and often sets the context for business process modeling and requirements analysis. These requirements often include:

- Data flow diagrams to illustrate how data flows through the information systems (different from flowcharts depicting algorithmic flow of business activities)

- Business process models and analysis using standard flowchart notations to depict business processes (as-is and to-be)

- Business context and background, including reasons for change

- Key business stakeholders requirements

- Logical data model and data dictionary references

Next step is identification of valid automation systems. Defining and documenting business requirements are necessary for this step. In step 3, according to automation systems, business model will become to system model. Step 4 identifies of candidate services. Candidate service is a service that satisfies an essential requirement, in whole or part. In a few cases, a candidate 
service may have applicability to more than one essential requirement. Next step decomposes the entire business process and review the details. After that, step 6 determines category of each services. These categories are three cases: business category, application category, and GIS category. In step 7, each service(s) decomposes to subservice(s) and in final step, each subservice(s) decomposes to one or more atomic service(s). Any atomic service(s) has the following characteristics:

- Separate from the higher level services

- No dependency to a specific organization; Any atomic service is able to employ in other organizations and has integrity

- These services are inseparable and so, they are in the lowest level of our platform

Figure 5 shows these eight steps of our service oriented analysis.

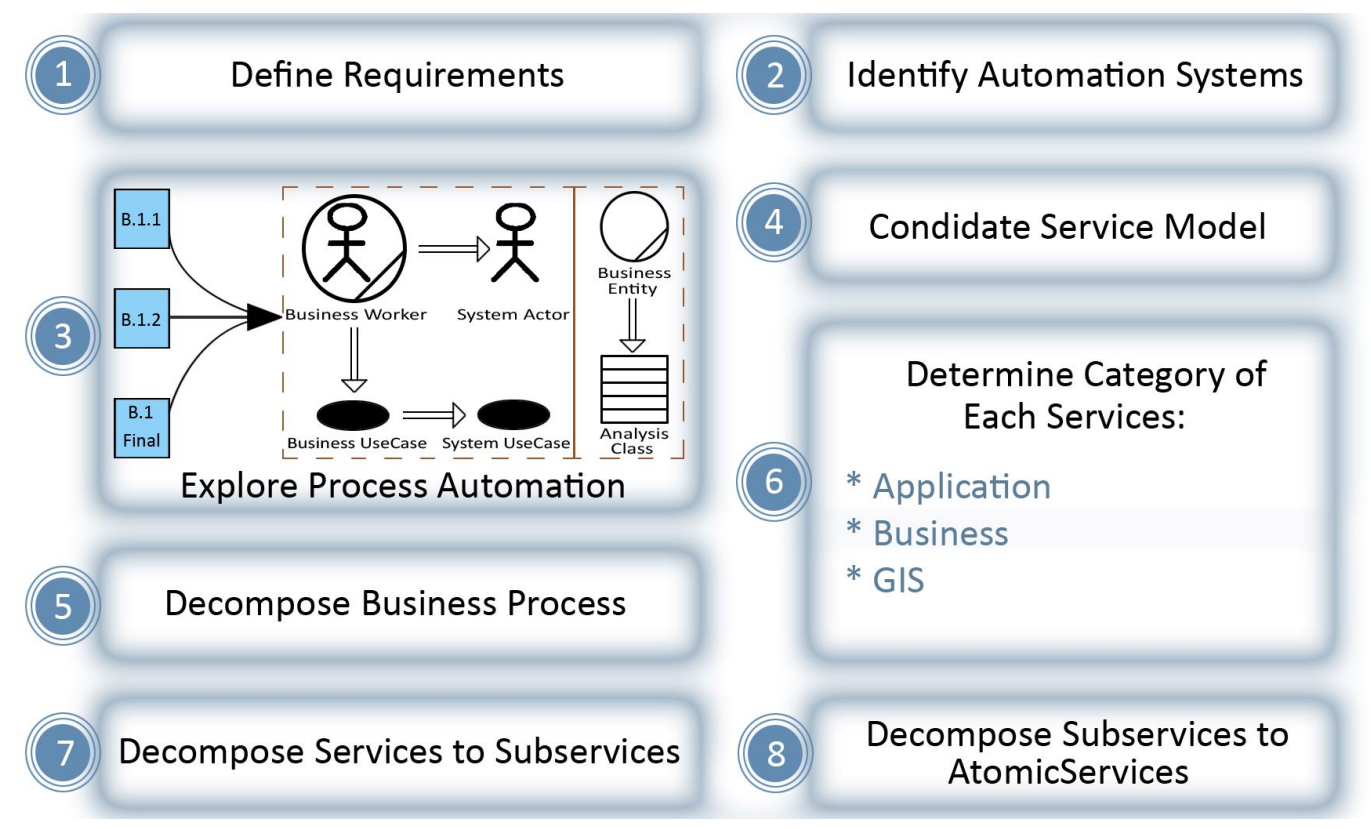

Figure 5. Service Oriented Analysis

\subsection{OUR Proposed Platform}

Our proposed platform, using service oriented architecture concepts and its advantages can increase business alignment, interoperability and agility in the organization. In this context, the proposed idea will be achieved by following two major steps: firstly, using service oriented advantages through classification of services in three levels which are implemented in the organization (GIS-based processes: category A \& GIS-independent processes: category B,C). As a result, business alignment, interoperability and agility will be increased. Secondly, introduces a dynamic interface and coordinator in order to establish a proper and mutual connection between independent categories of organization.

As shown in figure 6, our proposed platform has three categories and four levels. Category B and $\mathrm{C}$, are about GIS-independent processes and category A is related to GIS-based processes. As mentioned in previous section, GIS part of organization related to support, maintenance, and 
update spatial information. Levels of our platform have been classified from top to bottom. In higher level, we have an interface which is responsible for coordination between GIS-based processes and GIS-independent processes. Output of these processes, for update and / or insert in the geographic information system part, must send to this service.

As mentioned above, we have classified the organization services according to their complexity degree (decomposition ability), from top to bottom in level 3, level 2 and level 1 . We have put our main services in level 3. Main services in our proposed platform, are decomposable to lower levels of services. We have put these services in level 2 and called these services as "subservice". Similarly, these subservices can decompose to another services that we called these services as "atomicservice". Any atomicservice in our proposed approach has some features that are mentioned in previous section.

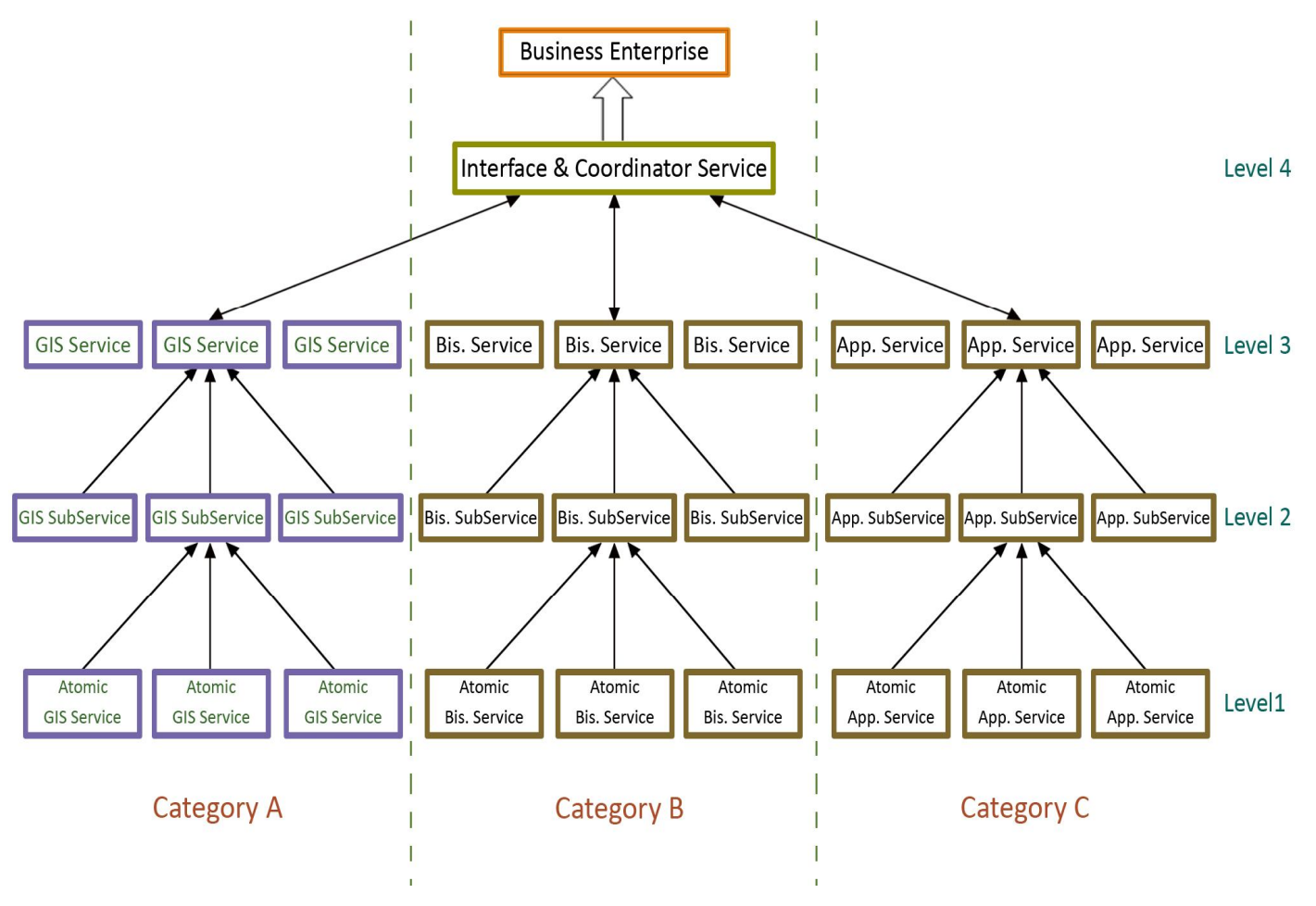

Figure 6. Our Proposed Platform

For example, suppose "Water and Sewage Company" that provides services to customers. A "water piping demand" is a service that will be in the third level of our proposed platform; This is a main service; It can decompose into other sub services such as "operation services", "request and coordinator services", "accounting services", and etc. These sub services will be classified in second level. Each of these sub services can decompose to other atomic services such as "fill the application form services", "pay services", and etc. These atomic services are independent from this organization. For example, "pay service" is a service that can be used in most organizations.

Note that, level one, two and three are linked together via communication messages and each level sends its output(s) to a higher level. At the end, and in order to update and synchronize the status between three parts, as noted above, after perform and apply a service in the organization, GIS-based processes and GIS-independent processes will be synchronize together through an interface and coordinator service. 
The presented platform, using service oriented architecture concepts has some advantages such as loose coupling, integrity, reusability, independency, and parallelism that encourages organizations to use it. In addition, using service oriented architecture in our platform, will result in better resource management in the organization. In more general view, this platform can used between two or more organizations in order to increasing interoperability, business alignment and agility between more than one organization. We called this as "infra-organization business alignment" and it can be in the future efforts.

\section{Conclusion And Plan For Future Works}

This paper showed that SOA as the best practice of the architectural concepts can be used for increasing business alignment in the enterprise applications. The proposed platform supports GIS functionalities along with enterprise viewpoints in a proper manner and will be useful for organizations wishing to develop enterprise applications with regards to GIS functionalities. This paper showed that SOA specifications such as loose coupling, reusability, abstraction, agility, and etc., can spread to organizations that using geographic information systems and increase their efficiency.

Future works of this study can be a web-based architecture that combines SOA and RIA (Rich Internet Application) for geographic information representation and processing. RIA is proposed by Macromedia in 2001 [30] in order to meet consumers' requirements like comprehensive information change, user experience requirements and etc., which changes the traditional requestresponse mechanism to a dynamic and asynchronous design pattern. RIA client and server have an asynchronous communication and so can reduce data transmission.

\section{REFERENCES}

[1] Fabling. Ty, "Enterprise GIS Architecture", ESRI Enterprise Consulting Group, Federal User Conference 2008.

[2] Murphy. Jonathan, Zwicker. Colin, "Geodatabase Essentials Part I", Esri International User Conference, San Diego, CA, july 12-16, 2010.

[3] Pierce. Brent, MacDougall. Gary, "Geodatabase Essentials Part II", Esri International User Conference, San Diego, CA,july 13-16, 2010.

[4] James B. Pick, "Geo-business GIS in the digital organization", Canada, 2008.

[5] Lu-hong. Zhou, Zeng-lin. Hong, "Study on Technology of GIS Applied to City Planning", 2011.

[6] Ye. chen, Yue. Wei, "The application of Geographical Information Technology and City Planning", PP.33-39, Dec 2007.

[7] H. M. Chen, "Towards Service Engineering: Service Orientation and Business-IT Alignment", 41st Hawaii International Conference on System Sciences (HICSS), 2008.

[8] Ma. Sai, Li. Minruo, Du. Weichang, "Service Composition for GIS", Congress on Services, 2008.

[9] Anand. Sriram, Padmanabhuni. Srinivas, Ganesh. Jai, "Perspectives on Service Oriented Architecture", Conference on Services Computing, 2005.

[10] Valipour. Mohammad Hadi, Amirzafari. Bavar, Niki Maleki. Khashayar, Daneshpour. Negin, "A Brief Survey of Software Architecture Concepts and Service Oriented Architecture", 2009. 
International Journal of Web \& Semantic Technology (IJWesT) Vol.3, No.1, January 2012

[11] OGC, http://www.opengeospatial.org/

[12] Amini. A, "GIS Software Architecture Based on SOA Concept and OGC Standards", 2nd international conference on computer engineering and technology (ICCET 2010), in press

[13] Shengjun. X, Liang. Z, "Developing GIS Software with component", International Symposium on Intelligent Information Technology Application Works, 2008.

[14] Tan. X, Feng. X, "Design of GIS Component Software with Example", First International Conference on Intelligent Networks and Intelligent Systems, 2008.

[15] Lu. X, "An Investigation on Service-Oriented Architecture for Constructing Distributed Web GIS Application", Proceedings of the 2005 IEEE International Conference on Services Computing (SCC 05), 2005.

[16] Kim. D.H., Kim2. K.S., Choz. H, Lee. J.H., "The Design and Implementation of Open GIS Service Component ", 2001.

[17] Shujun. D, Liang. L, Chengqi. C, "Research on Geographical Information Service Based on SOA", Proceedings of the IEEE International Conference on Automation and Logistics Qingdao, China September, 2008.

[18] Rui-sheng. J, Yan. J, Hong-mei. S, Xi-juan. W, "Research on Distributed GIS Process Modeling and Integration", Proceedings of 2008 IEEE International Symposium on IT in Medicine and Education, 2008 .

[19] Xiang. F, Ling. G, Jin. Y, "User-driven GIS Software Reuse Solution Based on SOA and Web2.0 Concept ", 2009.

[20] G. A. Lewis, E. Morris, S. Simanta, and L. Wrage, "Common Misconceptions about Service-Oriented Architecture," in Commercial-off-the-Shelf (COTS)- Based Software Systems,2007. ICCBSS '07. Sixth International IEEE Conference on,2007, pp. 123-130.

[21] A. D. a. P. Narasimhan, "Dependable Service- Oriented Computing," IEEE Internet Computing, March/April 2009, pp.11-15, 2009.

[22] R. K. Philip Bianco, Paulo Merson, "Evaluation of Service-Oriented Architecture", Software Engineering Institute/ Carnegie Mellon, vol. Technical Report, CMU/SEI-2007-TR-015, September 2007, 2007.

[23] Ghafari. M, Saleh. M, Modiri. N, "A Model to Increase the Trust in Service Oriented Architecture", Fifth Asia Modelling Symposium, 2011.

[24] M. Ibrahim, and G. Long, "Service Oriented Architecture and Enterprise Architecture", IBM developer Works, IBM Corporation, May 2007.

[25] Morita, Isao, "Toward Realization of Service-Oriented Architecture (SOA) ", July 2006.

[26] Erl. Thomas, "Service-Oriented Architecture: Concepts, Technology, and Design", Prentice Hall PTR Upper Saddle River, NJ, USA, 2005.

[27] J. Howells, B. Tether, and C. Europea, "Innovation in Services: Issues at Stake and Trends", Office for Official Publications of the European Communities, 2006.

[28] Fareghzadeh, Nafise, "Service Identification Approach to SOA Development", International Journal of Human and Social Sciences, 2009. 
International Journal of Web \& Semantic Technology (IJWesT) Vol.3, No.1, January 2012

[29] Shirazi H.M, Fareghzadeh N. and Seyyedi A, "A Combinational Approach to Service Identification in SOA", Journal of Applied Sciences Research. 2009.

[30] Jeremy. Allaire, "Macromedia Flash MX-A next-generation rich client", pp 1-4, 2002.

\section{Authors}

M. Saleh received his B.Sc degree in Computer Engineering from Payame Noor University, Iran in 2008. Currently he is pursuing his master degree in the Software Engineering in Payame Noor University, Tehran, Iran. His interests include Service Oriented Architecture, Information Systems, Security, and Coordination Problems.

Dr.T. Yaghoobi is an Assistant Professor at the Department of Computer and Information Technology Engineering in the Payame Noor University, Isfahan, Iran. She has received the PhD degree in Computer Sciences from Sheffield Hallam University, UK. Her main research interest is artificial intelligence.

Dr.A. Faraahi received the $\mathrm{PhD}$ degree in Computer Sciences from Bradford University. He has been working as a full-time faculty member, Assistant Professor and Head of Computer and Information Technology Department in the Payame Noor University. His research interests are programming especially that of educational systems. 